MATHEMATICS OF COMPUTATION

Volume 70, Number 235, Pages 1205-1220

S $0025-5718(00) 01243-6$

Article electronically published on June 12, 2000

\title{
BOUNDS ON DIFFERENCES OF ADJACENT ZEROS OF BESSEL FUNCTIONS AND ITERATIVE RELATIONS BETWEEN CONSECUTIVE ZEROS
}

\author{
JAVIER SEGURA
}

\begin{abstract}
Bounds for the distance $\left|c_{\nu, s}-c_{\nu \pm 1, s^{\prime}}\right|$ between adjacent zeros of cylinder functions are given; $s$ and $s^{\prime}$ are such that $\left.\nexists c_{\nu, s^{\prime \prime}} \in\right] c_{\nu, s}, c_{\nu \pm 1, s^{\prime}}[$; $c_{\nu, k}$ stands for the $k$ th positive zero of the cylinder (Bessel) function $\mathcal{C}_{\nu}(x)=$ $\cos \alpha J_{\nu}(x)-\sin \alpha Y_{\nu}(x), \alpha \in[0, \pi[, \nu \in \mathbb{R}$.

These bounds, together with the application of modified (global) Newton methods based on the monotonic functions $f_{\nu}(x)=x^{2 \nu-1} \mathcal{C}_{\nu}(x) / \mathcal{C}_{\nu-1}(x)$ and $g_{\nu}(x)=-x^{-(2 \nu+1)} \mathcal{C}_{\nu}(x) / \mathcal{C}_{\nu+1}(x)$, give rise to forward $\left(c_{\nu, k} \rightarrow c_{\nu, k+1}\right)$ and backward $\left(c_{\nu, k+1} \rightarrow c_{\nu, k}\right)$ iterative relations between consecutive zeros of cylinder functions.

The problem of finding all the positive real zeros of Bessel functions $\mathcal{C}_{\nu}(x)$ for any real $\alpha$ and $\nu$ inside an interval $\left[x_{1}, x_{2}\right], x_{1}>0$, is solved in a simple way.
\end{abstract}

\section{INTRODUCTION}

The positive zeros of cylinder functions have many applications in physics, technology and applied mathematics. Let us denote by $c_{\nu, s}$ the $s$ th positive zero of a cylinder function

$$
\mathcal{C}_{\nu}(x)=\cos \alpha J_{\nu}(x)-\sin \alpha Y_{\nu}(x),
$$

which satisfies the recurrence relations

$$
\begin{aligned}
& \mathcal{C}_{\nu+1}(x)+\mathcal{C}_{\nu-1}(x)=\frac{2 \nu}{x} \mathcal{C}_{\nu}(x), \\
& \mathcal{C}_{\nu}^{\prime}(x)=\left(\mathcal{C}_{\nu-1}(x)-\mathcal{C}_{\nu+1}(x)\right) / 2 .
\end{aligned}
$$

Several approximations exist to evaluate the zeros of Bessel functions of the first and second kinds $J_{\nu}(x)$ and $Y_{\nu}(x)$. In particular, McMahon's and Olver's asymptotic expansions and Olver's uniform asymptotic expansions (1, 14, 11, 26]) can be used to obtain zeros for large $\nu$ and/or large $s$. Series expansions [17, rational approximations [15], Chebyshev series expansions [16], and further asymptotic expansions [2, 5] have been developed which can serve as complement to McMahon's and Olver's asymptotic expansions. Additional information on monotonicity and convexity properties of the zeros, bounds for the first zeros of Bessel functions, and

Received by the editor January 7, 1999 and, in revised form, June 28, 1999.

2000 Mathematics Subject Classification. Primary 33C10.

Key words and phrases. Bessel functions, cylinder functions, adjacent and consecutive zeros, global Newton method. 
Sturm's inequalities can be found in [3, 4, 6, 7, 12, 13, and in previous publications by the same authors.

Several methods have been developed for the evaluation of the zeros of Bessel functions, among them:

1. N. M. Temme developed an ALGOL 60 computer program to evaluate the zeros of $J_{\nu}(x), Y_{\nu}(x), J_{\nu}^{\prime}(x)$ and $Y_{\nu}^{\prime}(x)$ for positive orders. Several asymptotic and uniform asymptotic expansions [26, 1, 23] were considered to obtain first approximations to the roots which were refined by applying a second order Newton iteration [22]. A Fortran 77 version of this algorithm can be found in [8].

2. R. Piessens presented a software package for the computation of the zeros of $J_{\nu}(x)$ for orders $\nu>-1$ and its turning points for positive orders [18. These programs use Chebyshev expansions [16] and asymptotic expansions for the zeros and turning points.

3. M. N. Vrahatis et al. developed the code RFSNFS [25] which uses the concept of topological degree to isolate the real zeros of the Bessel functions $J_{\nu}(x)$ and $Y_{\nu}(x)$ and their derivatives; a modified bisection rule is applied to evaluate the zeros and turning points for real and positive orders. In addition, a method to evaluate the complex zeros for real orders has been developed by the same group [9].

4. A novel approach to evaluate the zeros of Bessel functions was considered in [20]. A global Newton method convergent for cylinder functions $\mathcal{C}_{\nu}(x)=$ $\cos \alpha J_{\nu}(x)-\sin \alpha Y_{\nu}(x)$ was introduced. The Newton iteration based on the monotonic function $f_{\nu}(x)=x^{2 \nu-1} \mathcal{C}_{\nu}(x) / C_{\nu-1}(x)$ was proven to be convergent for any real values of $\nu$ and $\alpha$ and for any starting values $x_{0}>0$. The function $f_{\nu}(x)$ is similar to the trigonometric function $\tan x$ in its monotonicity and convexity properties. In this way, given a real starting value $x_{0}>0$ lying inside some branch of $f_{\nu}(x)$, the Newton iteration defined in [20] converges to the zero of $C_{\nu}(x)$ in this same branch (if any).

By using this global Newton method, complemented with rough (but sufficiently accurate) first approximations to the roots, codes to evaluate the zeros of $J_{\nu}(x)$ in intervals $\left[x_{\min }, x_{\max }\right], x_{\min }>0$, were built [21]. Such algorithms proved to be particularly efficient for the evaluation of the first positive zeros for moderate orders (positive or negative).

In this paper, a simple and easy to implement procedure for finding the positive real zeros of any real solution of equations 0.2 is presented. The method gives with certainty all the positive roots of the equation $\cos \alpha J_{\nu}(x)-\sin \alpha Y_{\nu}(x)=0$ inside any given interval $\left[x_{\min }, x_{\max }\right], x_{\min }>0$, for any real $\alpha$ and $\nu$.

The following ingredients will be taken into account in order to build this procedure:

1. Two globally convergent Newton methods based on the functions $f_{i, \nu}(x)=$ $-i x^{2 i \nu-1} \mathcal{C}_{\nu}(x) / \mathcal{C}_{\nu+i}(x), i= \pm 1$, which generalize the Newton iteration presented in [20].

2. Bounds for differences of adjacent zeros $\left|c_{\nu, s}-c_{\nu \pm 1, s^{\prime}}\right|$, with $s, s^{\prime}$ such that $\left.\nexists c_{\nu, s^{\prime \prime}} \in\right] c_{\nu, s}, c_{\nu \pm 1, s^{\prime}}[$.

3. Forward $\left(c_{\nu, k} \rightarrow c_{\nu, k+1}\right)$ and backward $\left(c_{\nu, k+1} \rightarrow c_{\nu, k}\right)$ iterative relations between consecutive zeros of cylinder functions $\mathcal{C}_{\nu}(x)$ which are based on the 
two globally convergent Newton iterations and the bounds on differences of adjacent zeros.

Numerical examples will illustrate the method to find the positive roots of $C_{\nu}(x)$ inside real intervals.

\section{Global Newton methods}

In this section, two globally convergent Newton iterations based on the monotonic functions

$$
f_{i, \nu}(x)=-i x^{-2 i \nu-1} C_{\nu}(x) / C_{\nu+i}(x), i= \pm 1
$$

will be built. First, the concepts of consecutive and adjacent zeros of Bessel functions are introduced. Then the monotonicity and convexity properties of $f_{+1, \nu}(x)$ are proved to follow immediately from those already shown for $f_{-1, \nu}(x)=$ $x^{2 \nu-1} C_{\nu}(x) / C_{\nu-1}(x)[20]$.

1.1. Consecutive and adjacent zeros. It is a well known fact that cylinder functions $\mathcal{C}_{\nu}(x)$ have an infinite and countable number of positive real zeros and that all such zeros are simple. Besides, the zeros of Bessel functions of consecutive orders are interlaced:

Theorem 1.1 (Interlacing). Between two consecutive positive zeros of $\mathcal{C}_{\nu+1}(x)$, there is one and only one zero of $\mathcal{C}_{\nu}(x)$ and, conversely, between two consecutive positive zeros of $\mathcal{C}_{\nu}(x)$ there is exactly one zero of $\mathcal{C}_{\nu+1}(x)$.

Notation 1.2. We adopt the standard notation $c_{\nu, s}$ for the $s$ th positive zero of a Bessel function $\mathcal{C}_{\nu}(x) . \mathcal{Z}_{\nu}$ will denote the set of positive zeros of a cylinder function $\mathcal{C}_{\nu}$. Similarly, $\mathcal{Z}_{\nu+i}$ is the set of positive zeros of $\mathcal{C}_{\nu+i}(x)$ with $i=+1$ or $i=-1$.

The zeros $c_{\nu, s}$ and $c_{\nu, s+1}$ are, for obvious reasons, said to be consecutive.

A zero $c_{\nu \pm 1, s^{\prime}}$ which lies between two consecutive zeros $c_{\nu, s}$ and $c_{\nu, s+1}$, will be said to be adjacent to them (and vice versa). In other words:

Definition 1.3 (Adjacent zeros of $\mathcal{C}_{\nu}(x)$ and $\mathcal{C}_{\nu \pm 1}(x)$ ). Let $c_{\nu, s}$ and $c_{\nu^{\prime}, s^{\prime}}$ be such that $c_{\nu, s}<c_{\nu^{\prime}, s^{\prime}}$, and let $\nu^{\prime}=\nu+1$ or $\nu^{\prime}=\nu-1 . c_{\nu, s}$ and $c_{\nu^{\prime}, s^{\prime}}$ are adjacent zeros $\Leftrightarrow \mathcal{C}_{\nu}(x) \neq 0$ and $\left.\mathcal{C}_{\nu^{\prime}}(x) \neq 0 \forall x \in\right] c_{\nu, s}, c_{\nu^{\prime}, s^{\prime}}[$.

Given Theorem 1.1, there is one and only one zero $c_{\nu, s}$ in each interval ]$\left.c_{\nu^{\prime}, s^{\prime}}, c_{\nu^{\prime}, s^{\prime}+1}\right], \nu^{\prime}=\nu \pm 1$. Thus, the following definition can be considered:

Definition $1.4\left(B_{i}\left(c_{\nu, s}\right)\right.$ : branch containing $\left.c_{\nu, s}\right)$. Let $i=+1$ or $i=-1$.

1. If $\left.\left.c_{\nu, s} \in\right] c_{\nu+i, s^{\prime}}, c_{\nu+i, s^{\prime}+1}\right]$, we write $\left.\left.B_{i}\left(c_{\nu, s}\right) \equiv\right] c_{\nu+i, s^{\prime}}, c_{\nu+i, s^{\prime}+1}\right]$.

2. If $c_{\nu+i, 1}<c_{\nu, 1}$, we write $\left.\left.B_{i}\left(0_{\nu}\right) \equiv\right] 0, c_{\nu+i, 1}\right]$.

3. If $c_{\nu, 1}<c_{\nu+i, 1}$, we write $\left.\left.B_{i}\left(c_{\nu, 1}\right) \equiv\right] 0, c_{\nu+i, 1}\right]$ and $B_{i}\left(0_{\nu}\right)=\emptyset$.

Remark 1.5. Considering Theorem 1.1, it becomes obvious that

$$
\begin{gathered}
\mathbb{R}^{+}=B_{i}\left(0_{\nu}\right) \cup\left(\bigcup_{s=1}^{\infty} B_{i}\left(c_{\nu, s}\right)\right) ; B_{i}\left(0_{\nu}\right) \cap B_{i}\left(c_{\nu, s}\right)=\emptyset \forall s \in \mathbb{N} ; \\
B_{i}\left(c_{\nu, s}\right) \cap B_{i}\left(c_{\nu, s^{\prime}}\right)=\emptyset \Longleftrightarrow s \neq s^{\prime}, s, s^{\prime} \in \mathbb{N} .
\end{gathered}
$$


Let us now state a well known theorem on the distance between consecutive zeros [23]. Theorem 1.6] will be useful in order to prove several results in Sections 3 and 4. Later (Section 2), bounds on the distance between adjacent zeros will be established.

Theorem 1.6 (Bounds for differences of consecutive zeros). Let $c_{\nu, s}, c_{\nu, s+1}$ be two positive zeros of $\mathcal{C}_{\nu}(x)$.

1. If $|\nu| \neq 1 / 2$, then $\left(c_{\nu, s+1}-c_{\nu, s}-\pi\right)(|\nu|-1 / 2)>0$.

2. If $|\nu|=1 / 2$, then $c_{\nu, s+1}-c_{\nu, s}-\pi=0$.

Theorem 1.6 can be proved using Sturm's comparison theorem [23].

1.2. The monotonic functions $f_{ \pm 1, \nu}(x)$. In reference[20], the function $f_{-1, \nu}(x)$ (equation 1.1) was shown to be monotonic in the positive real axis except at the zeros of $\mathcal{C}_{\nu-1}(x)\left(\mathcal{Z}_{\nu-1}\right)$. A global Newton method to find the zeros of cylinder functions $\mathcal{C}_{\nu}(x)$ for any real order $\nu$ was built. That method was based on the monotonicity and convexity properties of $f_{-1, \nu}(x)$.

Let us show that a second global Newton algorithm can be built based on the function $f_{+1, \nu}(x)=-x^{-2 \nu-1} \mathcal{C}_{\nu}(x) / \mathcal{C}_{\nu+1}(x)$. First, we define in compact form the functions under study (keeping temporarily the angle $\alpha$ in the notation):

Definition 1.7. Letting $\mathcal{C}_{\nu}(x, \alpha)=\cos \alpha J_{\nu}(x)-\sin \alpha Y_{\nu}(x)$ and $i=+1$ or $i=-1$, we define

$$
\begin{gathered}
H_{i, \nu}(x, \alpha)=-i \mathcal{C}_{\nu}(x, \alpha) / \mathcal{C}_{\nu+i}(x, \alpha), \\
f_{i, \nu}(x, \alpha)=x^{-\delta_{i}(\nu)} H_{i, \nu}(x, \alpha), \\
\delta_{i}(\nu)=2 i \nu+1 .
\end{gathered}
$$

The following relations can be proved with ease.

Lemma 1.8. $H_{-1,-\nu}(x, \alpha)=H_{+1, \nu}(x, \alpha+\nu \pi) ; f_{-1,-\nu}(x, \alpha)=f_{+1, \nu}(x, \alpha+\nu \pi)$.

Proof. Use the reflection formula $\mathcal{C}_{-\nu}(x, \alpha)=\mathcal{C}_{\nu}(x, \alpha+\nu \pi)$ [20].

Remark 1.9. We will use the notation

$$
\mathcal{C}_{\nu}(x) \equiv \mathcal{C}_{\nu, \alpha}(x), H_{i, \nu}(x) \equiv H_{i, \nu}(x, \alpha), f_{i, \nu}(x) \equiv f_{i, \nu}(x, \alpha)
$$

for any fixed $\alpha$ and $i= \pm 1$.

Lemma 1.8 demonstrates that the monotonicity and convexity properties of the functions $H_{-1, \nu}(x)$ and $f_{-1, \nu}(x)$ have their counterpart for $H_{+1, \nu}(x)$ and $f_{+1, \nu}(x)$. Lemma 1.10 generalizes some of the results presented in 20].

Lemma 1.10. Let $i=+1$ or $i=-1$.

1. $H_{i, \nu}(x), f_{i, \nu}(x)$ and their derivatives are continuous functions in $\mathbb{R}^{+} \backslash \mathcal{Z}_{\nu+i}$.

2. $H_{i, \nu}(x)$ has an infinite and countable number of positive zeros $\left(\mathcal{Z}_{\nu}\right)$ and singularities $\left(\mathcal{Z}_{\nu+i}\right)$. The zeros and singularities are interlaced. The same holds for $f_{i, \nu}(x)$.

3. $H_{i, \nu}^{\prime}(x)=1+H_{i, \nu}(x)^{2}+\delta_{i}(\nu) H_{i, \nu}(x) / x \forall x \in \mathbb{R}^{+} \backslash \mathcal{Z}_{\nu+i}$.

4. $f_{i, \nu}^{\prime}(x)=x^{-\delta_{i}(\nu)}\left(1+H_{i, \nu}(x)^{2}\right) \forall x \in \mathbb{R}^{+} \backslash \mathcal{Z}_{\nu+i}$.

5. $H_{i, \nu}(x), i= \pm 1$ is strictly increasing for $x>\min \left(c_{\nu, 1}, c_{\nu+i, 1}\right), x \in \mathbb{R}^{+} \backslash \mathcal{Z}_{\nu+i}$.

6. $f_{i, \nu}(x), i= \pm 1$ is strictly increasing in $\mathbb{R}^{+} \backslash \mathcal{Z}_{\nu+i}$. 
The proof of Lemma 1.10 is omitted because all the properties described were demonstrated for $i=-1$ in [20]. The generalization to $i= \pm 1$ becomes straightforward by taking into account Lemma 1.8 .

Considering Lemma 1.10 parts 2 and 5, the next corollary follows.

Corollary 1.11. If $x \in B_{i}\left(c_{\nu, s}\right), x \neq c_{\nu, s}$, then $\left(x-c_{\nu, s}\right) H_{i, \nu}(x)>0$.

This result together with the formula for $H_{i, \nu}^{\prime}(x)$ (Lemma 1.10) will be used to derive bounds on differences of adjacent zeros.

1.3. Global convergence of the Newton iterations. Global Newton methods can be built based on the functions $f_{i, \nu}(x), i= \pm 1$. In order to generalize the global Newton method presented in reference [20, one only needs to replace $H_{\nu}(x)$ ( $\left.\equiv H_{-1, \nu}(x)\right)$ by $H_{i, \nu}(x)$ in Theorem 2.1 of [20]. The functions $\mathcal{T}_{i, \nu}(x)$ resulting from such replacement (Definition 1.12) constitute a pair of global Newton iterations (Theorem 1.13).

Definition 1.12 (Globally convergent Newton iterations $\mathcal{T}_{i, \nu}(x), i= \pm 1$ ).

$$
\begin{aligned}
& \mathcal{T}_{i, \nu}(x)=\Theta\left(\bar{T}_{i, \nu}(x)\right) \bar{T}_{i, \nu}(x)+\Theta\left(-\bar{T}_{i, \nu}(x)\right) \alpha x, \quad 0<\alpha<1, \\
& \Theta(y)=1 \quad \text { for } y>0, \quad \Theta(y)=0 \quad \text { for } y \leq 0, \\
& \bar{T}_{i, \nu}(x)=x-\bar{S}_{i, \nu}(x), \quad \bar{S}_{i, \nu}(x)=\frac{\bar{H}_{i, \nu}(x)}{1+\bar{H}_{i, \nu}(x)^{2}}, \\
& \bar{H}_{i, \nu}(x)=\left\{\begin{array}{l}
\frac{H_{i, \nu}(x)}{\left|H_{i, \nu}(x)\right|} \min \left(1,\left|H_{i, \nu}(x)\right|\right), x \notin \mathcal{Z}_{\nu+i}^{\mathcal{C}}, \\
1, x \in \mathcal{Z}_{\nu+i}^{\mathcal{C}} .
\end{array}\right.
\end{aligned}
$$

Theorem 1.13 (Global convergence). Let us denote by $\mathcal{T}_{i, \nu}^{(n)}\left(x_{0}\right)$ the nth iteration of $\mathcal{T}_{i, \nu}$ over a starting value $x_{0}>0$. Then $\exists \lim _{n \rightarrow \infty} \mathcal{T}_{i, \nu}^{(n)}\left(x_{0}\right), \forall x_{0}>0, \forall \nu \in$ $\mathbb{R}, i= \pm 1$ and

1. If $x_{0} \in B_{i}\left(c_{\nu, s}\right)$, then $\lim _{n \rightarrow \infty} \mathcal{T}_{i, \nu}^{(n)}\left(x_{0}\right)=c_{\nu, s}$.

2. If $x_{0} \in B_{i}\left(0_{\nu}\right)$, then $\lim _{n \rightarrow \infty} \mathcal{T}_{i, \nu}^{(n)}\left(x_{0}\right)=0$.

Remark 1.14. By taking into account Remark 1.5 one observes that given any $x_{0}>$ 0 there is one and only one branch $B_{i}\left(c_{\nu, s}\right)$ (or $B_{i}\left(0_{\nu}\right)$ ) such that $x_{0} \in B_{i}\left(c_{\nu, s}\right)$ (or $x_{0} \in B_{i}\left(0_{\nu}\right)$ ). Thus, by virtue of Theorem [1.13] and given $x_{0}>0$, either $\lim _{n \rightarrow \infty} \mathcal{T}_{i, \nu}^{(n)}\left(x_{0}\right)=c_{\nu, s}$ for some $s$ or $\lim _{n \rightarrow \infty} \mathcal{T}_{i, \nu}^{(n)}\left(x_{0}\right)=0$. Convergence to 0 will only take place when $\left.\left.x_{0} \in\right] 0, c_{\nu+i, 1}\right]$ and $c_{\nu+i, 1}<c_{\nu, 1}$, that is, when $x_{0} \in B_{i}\left(0_{\nu}\right) \neq \emptyset$ (see Definition 1.4).

\section{Bounds on the Differences $\left|c_{\nu, s}-c_{\nu \pm 1, s^{\prime}}\right|$ BetWeen AdJACENT Zeros}

The iteration of $\mathcal{T}_{i, \nu}$ converges to $c_{\nu, s}$ (Theorem 1.13) for any a starting value $x_{0} \in B_{i}\left(c_{\nu, s}\right)$. In Section 3, we will describe how to evaluate $c_{\nu, s \pm 1}$ once $c_{\nu, s}$ is known. Bounds on the differences of adjacent zeros are required in order to build such (iterative) relations between $c_{\nu, s}$ and $c_{\nu, s \pm 1}$. 
Theorem 2.1 (Bounds for differences of adjacent zeros). Let $c_{\nu, s}, c_{\nu+i, s^{\prime}}, i= \pm 1$, be adjacent zeros. Let $\delta_{i}(\nu)=2 i \nu+1$.

1. If $\delta_{i}(\nu) \neq 0$, then $\delta_{i}(\nu)\left(c_{\nu+i, s^{\prime}}-c_{\nu, s}\right)\left[\pi / 2-\left|c_{\nu+i, s^{\prime}}-c_{\nu, s}\right|\right]>0$.

2. If $\delta_{i}(\nu)=0$, then $\left|c_{\nu+i, s^{\prime}}-c_{\nu, s}\right|=\pi / 2$.

Proof. Let $\delta_{i}(\nu) \neq 0$ (the case $\delta_{i}(\nu)=0$ is trivial). From Lemma 1.10 we know that

$$
H_{i, \nu}^{\prime}(x)=1+H_{i, \nu}(x)^{2}+\delta_{i}(\nu) H_{i, \nu}(x) / x .
$$

Equation 2.1 can be proved by deriving $H_{i, \nu}(x)$ (equations 1.2) with respect to $x$ and employing the recurrences (equation 0.2 ) to rewrite the derivative in terms of $H_{i, \nu}(x)$. From this equation,

$$
\delta_{i}(\nu) H_{i, \nu}(x)\left[\frac{H_{i, \nu}^{\prime}(x)}{1+H_{i, \nu}(x)^{2}}-1\right]=\frac{\delta_{i}^{2}(\nu) H_{i, \nu}(x)^{2}}{x\left(1+H_{i, \nu}(x)^{2}\right)} \quad \forall x \in \mathbb{R}^{+} \backslash \mathcal{Z}_{\nu+i}
$$

and then

$$
\delta_{i}(\nu) H_{i, \nu}(x)\left[\frac{H_{i, \nu}^{\prime}(x)}{1+H_{i, \nu}(x)^{2}}-1\right]>0 \quad \forall x \in \mathbb{R}^{+} \backslash \mathcal{Z}_{\nu} \bigcup \mathcal{Z}_{\nu+i} .
$$

In particular, the inequality holds $\forall x \in B_{i}^{\circ}\left(c_{\nu, s}\right) \backslash c_{\nu, s}$ and for any given $s \in \mathbb{N}$. $B_{i}^{\circ}\left(c_{\nu, s}\right)$ denotes the interior of $B_{i}\left(c_{\nu, s}\right)$.

Considering Corollary 1.11 we have

$$
h_{i, \nu}(x) \equiv \delta_{i}(\nu) \operatorname{sign}\left[x-c_{\nu, s}\right]\left[\frac{H_{i, \nu}^{\prime}(x)}{1+H_{i, \nu}(x)^{2}}-1\right]>0 \quad \forall x \in B_{i}^{\circ}\left(c_{\nu, s}\right) \backslash\left\{c_{\nu, s}\right\}
$$

and then for all $x^{\prime} \in B_{i}^{\circ}\left(c_{\nu, s}\right) \backslash c_{\nu, s}$,

$$
\begin{aligned}
\operatorname{sign}\left[\int_{c_{\nu, s}}^{x^{\prime}} h_{i, \nu}(x) d x\right] & =\operatorname{sign}\left[x^{\prime}-c_{\nu, s}\right] \\
& \Rightarrow \operatorname{sign}\left[x^{\prime}-c_{\nu, s}\right] \int_{c_{\nu, s}}^{x^{\prime}} h_{i, \nu}(x) d x>0 .
\end{aligned}
$$

Writing $s_{i}\left(\nu, x^{\prime}\right) \equiv \delta_{i}(\nu)\left(x^{\prime}-c_{\nu, s}\right)$,

$$
\begin{aligned}
& s_{i}\left(\nu, x^{\prime}\right) \int_{c_{\nu, s}}^{x^{\prime}} \operatorname{sign}\left[x-c_{\nu, s}\right]\left(\frac{H_{i, \nu}^{\prime}(x)}{1+H_{i, \nu}(x)^{2}}-1\right) d x \\
& \quad=s_{i}\left(\nu, x^{\prime}\right) \operatorname{sign}\left[x^{\prime}-c_{\nu, s}\right] \int_{c_{\nu, s}}^{x^{\prime}}\left(\frac{H_{i, \nu}^{\prime}(x)}{1+H_{i, \nu}(x)^{2}}-1\right) d x>0 .
\end{aligned}
$$

By integrating we obtain

$$
s_{i}\left(\nu, x^{\prime}\right) \operatorname{sign}\left[x^{\prime}-c_{\nu, s}\right]\left(\arctan \left(H_{i, \nu}\left(x^{\prime}\right)\right)-\left(x^{\prime}-c_{\nu, s}\right)\right)>0,
$$

and by taking into account Corollary 1.11

$$
s_{i}\left(\nu, x^{\prime}\right)\left(\left|\arctan \left(H_{i, \nu}\left(x^{\prime}\right)\right)\right|-\left|x^{\prime}-c_{\nu, s}\right|\right)>0 \quad \forall x^{\prime} \in B_{i}^{\circ}\left(c_{\nu, s}\right) \backslash c_{\nu, s} .
$$

Taking now the limit $x^{\prime} \rightarrow c_{\nu+i, s^{\prime}}$, being $c_{\nu+i, s^{\prime}}$ adjacent to $c_{\nu, s}$, we have $H_{i, \nu}\left(x^{\prime}\right) \rightarrow \pm \infty$ and $s_{i}\left(\nu, c_{\nu+i, s^{\prime}}\right)\left(\pi / 2-\left|c_{\nu+i, s^{\prime}}-c_{\nu, s}\right|\right)>0$, and then

$$
\delta_{i}(\nu)\left(c_{\nu+i, s^{\prime}}-c_{\nu, s}\right)\left(\pi / 2-\left|c_{\nu+i, s^{\prime}}-c_{\nu, s}\right|\right)>0 \text {. }
$$




\section{Iterative RELAtions BetWeEN CONSECUtiVe ZeRos $c_{\nu, s}, c_{\nu, s \pm 1}$}

Once two global Newton iterations have been defined and bounds on differences of adjacent zeros have been given, iterative relations between consecutive zeros of $\mathcal{C}_{\nu}(x)$ can be established.

In this section, the main result is Theorem 3.6 part 2: once a zero $c_{\nu, s}$ is known, the starting value $c_{\nu, s} \pm \pi$ guarantees convergence to $c_{\nu, s \pm 1}$ by appropriately choosing one of the two Newton iterations.

First, it is shown that $c_{\nu, s} \pm \pi$ is inside $B_{i}\left(c_{\nu, s \pm 1}\right)$ either for $i=+1$ or $i=-1$ (there is a possible exception when $|\nu|<1 / 2$ ). This result (Lemma 3.5), together with the global convergence of the Newton iterations $\mathcal{T}_{i, \nu}(x)$ (Theorem 1.13) is enough to prove the main result (Theorem 3.6).

Notation 3.1. From now on $\operatorname{sign}(x)$ will denote the function $\operatorname{sign}(x)=+1$ if $x \geq 0$ and $\operatorname{sign}(x)=-1$ when $x<0$. The next results could have been stated in the same way had we chosen $\operatorname{sign}(0)=-1$.

Lemma 3.2. Let $|\nu|>1 / 2$. Let $i= \pm 1, s \in \mathbb{N}$ be such that $k \equiv s+\operatorname{sign}(i \nu)>0$. Then $c_{\nu, s}+\gamma \operatorname{sign}(i \nu) \in B_{i}\left(c_{\nu, k}\right) \forall \gamma \in[\pi / 2, \pi]$.

Proof. Let $c_{\nu, s}, c_{\nu+i, s^{\prime}}$ be adjacent zeros. Considering that $\operatorname{sign}\left(\delta_{i}(\nu)\right)=\operatorname{sign}(i \nu)$ when $|\nu|>1 / 2$, we have that (see Theorem 2.1)

1. If $i \nu>0,\left|c_{\nu+i, s^{\prime}}-c_{\nu, s}\right|<\pi / 2$ when $c_{\nu+i, s^{\prime}}>c_{\nu, s}$, then $c_{\nu, s}+\gamma>c_{\nu+i, s^{\prime}}$ for $\gamma \geq \pi / 2$.

2. If $i \nu<0,\left|c_{\nu+i, s^{\prime}}-c_{\nu, s}\right|<\pi / 2$ when $c_{\nu+i, s^{\prime}}<c_{\nu, s}$, then $c_{\nu, s}-\gamma<c_{\nu+i, s^{\prime}}$ for $\gamma \geq \pi / 2$.

Thus,

$$
x_{1} \equiv c_{\nu, s}+\gamma \operatorname{sign}(i \nu) \notin B_{i}\left(c_{\nu, s}\right) \text { for } \gamma \geq \pi / 2 .
$$

Now it is a simple matter to check that $x_{1} \in B_{i}\left(c_{\nu+i, k}\right)$ when $\gamma \leq \pi$, where $k=s+\operatorname{sign}(i \nu)$. From Theorem 1.6, it follows that $c_{\nu, s+1}-c_{\nu, s} \geq \pi \forall s \in \mathbb{N}$ (since $|\nu|>1 / 2$ ) and then $c_{\nu, s}+\gamma<c_{\nu, s+1}$ for $\gamma \leq \pi$. Similarly, $c_{\nu, s}-\gamma>c_{\nu, s-1}$ (when $s-1>0$ ). Thus, choosing $\pi / 2 \leq \gamma \leq \pi, x_{1}$ necessarily lies in a branch contiguous to $B_{i}\left(c_{\nu, s}\right)$. 1.6.

The following result, valid for $|\nu| \leq 1 / 2$, can be proved by considering Theorem

Lemma 3.3. Let $j= \pm 1$ and $s \in \mathbb{N}$ be such that $s+j>0$. If $|\nu| \leq 1 / 2$, then $c_{\nu, s}+j \pi \in B_{i}\left(c_{\nu, s+j}\right)$ both for $i=+1$ and $i=-1$ except, possibly, when $s+j=1$ and $c_{\nu, 1}<c_{\nu+i, 1}$.

Proof. $|\nu| \leq 1 / 2 \Rightarrow c_{\nu, n+1}-c_{\nu, n} \leq \pi$ and $c_{\nu+i, n^{\prime}+1}-c_{\nu+i, n^{\prime}} \geq \pi \forall n, n^{\prime} \in \mathbb{N}$. Thus given the interlacing properties (Lemma 1.1), the sequence of zeros

$$
c_{\nu, s}<c_{\nu+i, s^{\prime}+1}<c_{\nu, s+1}<c_{\nu+i, s^{\prime}+2}
$$

with $s \geq 0$, is such that $c_{\nu, s+1}<c_{\nu, s}+\pi<c_{\nu+i, s^{\prime}+2}$. In other words

$$
c_{\nu, s}+\pi \in B_{i}\left(c_{\nu, s+1}\right) \quad \forall s \in \mathbb{N} .
$$

Similarly, for $s \geq 3$ can write

$$
c_{\nu+i, s^{\prime}-1}<c_{\nu, s-1}<c_{\nu+i, s^{\prime}}<c_{\nu, s}
$$


and $c_{\nu+i, s^{\prime}}<c_{\nu, s}-\pi<c_{\nu, s-1}$, that is

$$
c_{\nu, s}-\pi \in B_{i}\left(c_{\nu, s-1}\right) \quad s \geq 3
$$

while for $s=2$, the first inequality $\left(c_{\nu+i, s^{\prime}-1}<c_{\nu, s-1}\right)$ can only be considered when $c_{\nu+i, 1}<c_{\nu, 1}$. This is why exceptions to Lemma 3.3 can be found when $s=2$ and $j=-1$ (and $\left.c_{\nu, 1}<c_{\nu+i, 1}\right)$.

Remark 3.4. When $|\nu|<1 / 2$ and $c_{\nu, 1}<c_{\nu+i, 1}$, it may happen that $c_{\nu, 1}<c_{\nu, 2} \leq \pi$ and $c_{\nu, 2}-\pi \leq 0$. This fact also explains the exception in Lemma 3.3 and in the next two results. Such exception never takes place for $|\nu| \geq 1 / 2$, since in this case, as we will later show, $c_{\nu, 2} \geq \pi$ (Lemma 4.6), and besides, $c_{\nu, s}-\pi>c_{\nu, s-1}$ for $s \geq 2$. This exception is inherited by Lemma 3.5 and Theorem 3.6. Theorem 4.4 will overcome this limitation.

By taking into account Lemmas 3.3 and 3.2 one can prove the following result.

Lemma 3.5. Let $j= \pm 1$ and $s \in \mathbb{N}$ be such that $s+j>0$. Then $c_{\nu, s}+j \pi \in$ $B_{\operatorname{sign}(j \nu)}\left(c_{\nu, s+j}\right)$ except, possibly, when $|\nu| \leq 1 / 2, s+j=1$ and $c_{\nu, 1}<c_{\nu+\operatorname{sign}(j \nu), 1}$.

Proof. For $|\nu|<1 / 2$, the result is a consequence of Lemma 3.3. The proof for $|\nu|>1 / 2$ follows from Lemma 3.2 for the particular case $\gamma=\pi$, since

$$
c_{\nu, s}+\operatorname{sign}(j \nu) \pi \in B_{j}\left(c_{\nu, s+\operatorname{sign}(j \nu)}\right) \Longleftrightarrow c_{\nu, s}+j \pi \in B_{\operatorname{sign}(j \nu)}\left(c_{\nu, s+j}\right) .
$$

Finally, the main result follows from Lemmas 3.3, 3.5] and Theorem 1.13,

Theorem 3.6 (Iterative relations between consecutive zeros). Let $j= \pm 1$ and $s \in$ $\mathbb{N}$ be such that $s+j>0$.

1. If $|\nu| \leq 1 / 2$, then $c_{\nu, s+j}=\lim _{n \rightarrow \infty} \mathcal{T}_{i, \nu}^{(n)}\left[c_{\nu, s}+j \pi\right]$ for $i=+1$ or $i=-1$, except, possibly, when $s+j=1$ and $c_{\nu, 1}<c_{\nu+i, 1}$.

2. $c_{\nu, s+j}=\lim _{n \rightarrow \infty} \mathcal{T}_{\operatorname{sign}(j \nu), \nu}^{(n)}\left[c_{\nu, s}+j \pi\right] \forall \nu \in \mathbb{R}$ except, possibly, when $|\nu|<1 / 2$, $s+j=1$ and $c_{\nu, 1}<c_{\nu+\operatorname{sign}(j \nu), 1}$.

\section{Algorithms to Find All the positive ReAl zeros IN AN INTERVAL $\left[x_{\min }, x_{\max }\right], x_{\min }>0$}

The iterative relations given by Theorem [3.6] enable us to build algorithms capable of finding all the zeros of any real cylinder function inside a given interval $\left[x_{\min }, x_{\max }\right]$, with $x_{\min }>0$. For example, taking $x_{1}=\lim _{n \rightarrow \infty} \mathcal{T}_{-\operatorname{sign}(\nu), \nu}^{(n)}\left(x_{\max }\right)$ and $x_{k}=\lim _{n \rightarrow \infty} \mathcal{T}_{-\operatorname{sign}(\nu), \nu}^{(n)}\left(x_{k-1}-\pi\right)$ for $k>1$, the series of zeros of $\mathcal{C}_{\nu}(x)$ in an interval $\left[x_{\min }, x_{\max }\right]$ can be generated in decreasing order. However, the exception in the results of Section 3 will have to be overcome in order to obtain with certainty $c_{\nu, 1}$ once $c_{\nu, 2}$ has been evaluated.

The zeros can also be generated in increasing order by using the forward iterative relations, starting from $x_{\text {min }}$. We will now prove that, for any $x_{\min }>0$ and any real values of $\nu$ and $\alpha, \lim _{n \rightarrow \infty} \mathcal{T}_{\operatorname{sign}(\nu), \nu}^{(n)}\left(x_{\min }\right)$ is a positive zero of $\mathcal{C}_{\nu}(x)$. This fact ensures that the forward sweep is always valid and that no a priori estimations for the zeros are needed to start this sweep.

Being the globally convergent Newton iteration, we know from Theorem 1.13 that $\lim _{n \rightarrow \infty} \mathcal{T}_{\operatorname{sign}(\nu), \nu}^{(n)}\left(x_{\min }\right)=c_{\nu, s}$ when $c_{\nu, s} \in B_{\operatorname{sign}(\nu)}\left(c_{\nu, s}\right)$. Thus, the only case 
in which the forward iteration may not to converge to a positive zero of $\mathcal{C}_{\nu}(x)$ is when $x_{\min } \in B_{\operatorname{sign}(\nu)}\left(0_{\nu}\right)$, which is the same as saying that $0<x_{\min }<c_{\nu+\operatorname{sign}(\nu), 1}<c_{\nu, 1}$; however, this situation cannot take place:

Theorem 4.1 (Ordering between $c_{\nu, 1}$ and $\left.c_{\nu+\operatorname{sign}(\nu), 1}\right)$. The following statements are equivalent and hold for any real values of $\nu$ and $\alpha$ :

1. $B_{\operatorname{sign}(\nu)}\left(0_{\nu}\right)=\emptyset$.

2. $c_{\nu, 1}<c_{\nu+1,1}$ if $\nu \geq 0$ and $c_{\nu, 1}<c_{\nu-1,1}$ if $\nu \leq 0$.

3. $\lim _{x \rightarrow 0^{+}} f_{\operatorname{sign}(\nu), \nu}(x)<0$.

Proof. Statements 1 and 2 are clearly equivalent, given Definition 1.4. On the other hand, since $f_{\operatorname{sign}(\nu), \nu}(x)$ is monotonic, we have that

$$
c_{\nu, 1}<c_{\nu+\operatorname{sign}(\nu)} \Leftrightarrow \lim _{x \rightarrow 0^{+}} f_{\operatorname{sign}(\nu), \nu}(x)<0 .
$$

Thus, 2 and 3 are also equivalent.

Given the symmetry between positive and negative orders (Lemma 1.8) all that remains to be proved is that

$$
\lim _{x \rightarrow 0^{+}} f_{+1, \nu}(x)<0 \quad \forall \nu \geq 0 .
$$

By noticing that, as $x \rightarrow 0^{+}[10]$,

$$
J_{0}(x) \simeq 1, \quad Y_{0}(x) \simeq-\frac{2}{\pi} \log \frac{2}{x}
$$

and

$$
J_{\nu}(x) \simeq \frac{x^{\nu}}{2^{\nu} \Gamma(1+\nu)}, \quad Y_{\nu} \simeq-\frac{2^{\nu} \Gamma(\nu)}{\pi x^{\nu}} \text { for } \nu>0
$$

and writing $\mathcal{C}_{\nu}(x)=\cos \alpha J_{\nu}(x)-\sin \alpha Y_{\nu}(x)$ we see that, when $x \rightarrow 0^{+}$,

$$
\begin{gathered}
f_{+1, \nu}(x) \simeq-2(\nu+1) x^{-2(\nu+1)} \quad \text { for } \quad \nu \geq 0 \text { and } \alpha=0, \\
f_{+1,0}(x) \simeq-\log \frac{2}{x} \quad \text { for } \nu=0 \text { and } \alpha \neq 0, \\
f_{+1, \nu}(x) \simeq-\frac{1}{2 \nu} x^{-2 \nu} \quad \text { for } \nu>0 \text { and } \alpha \neq 0 .
\end{gathered}
$$

Therefore $\lim _{x \rightarrow 0^{+}} f_{+1, \nu}(x)=-\infty$ for $\nu \geq 0 \forall \alpha$.

From Theorems 1.13 and 4.1 easily follows that the iteration $\mathcal{T}_{\operatorname{sign}(\nu), \nu}(x)$ always converges to a positive real zero of $C_{\nu}(x)$ for any starting value $x_{0}>0$ and, differently from $\mathcal{T}_{-\operatorname{sign}(\nu), \nu}(x)$, it will never converge to 0 . In particular, we have:

Corollary 4.2 (Iterative formula for the first zero of cylinder functions). The first positive real zero of any real cylinder function can be written as

$$
\left.c_{\nu, 1}=\lim _{n \rightarrow \infty} \mathcal{T}_{\operatorname{sign}(\nu), \nu}^{(n)}(x) \quad \forall x \in\right] 0, c_{\nu+\operatorname{sign}(\nu), 1}[.
$$

Given Corollary 4.2, a prescription to evaluate all the zeros of a given cylinder function $\mathcal{C}_{\nu}(x)$ inside an interval $\left[x_{\min }, x_{\max }\right]$ can be established:

Theorem 4.3 (Forward evaluation of the zeros). The zeros of any real cylinder function $\mathcal{C}_{\nu}(x)$ inside an interval $\left[x_{\min }, x_{\max }\right], x_{\min }>0$, can be evaluated in the following way: 
1. Evaluate $x_{1} \equiv \lim _{m \rightarrow \infty} \mathcal{T}_{\operatorname{sign}(\nu), \nu}^{(m)}\left(x_{\min }\right)$. Then $x_{1}=c_{\nu, s}$ for some integer $s>0$ provided there is at least one positive zero of $\mathcal{C}_{\nu}(x), c_{\nu, s^{\prime}}$, such that $c_{\nu, s^{\prime}} \geq x_{\min }$.

2. Given $c_{\nu, k}$ with $k \geq s$, use the forward iterative relation

$$
c_{\nu, k+1}=\lim _{m \rightarrow \infty} \mathcal{T}_{\operatorname{sign}(\nu), \nu}^{(m)}\left(c_{\nu, k}+\pi\right)
$$

to generate the sequence of zeros

$$
c_{\nu, s}<c_{\nu, s+1}<\cdots<c_{\nu, s+n} \leq x_{\max }<c_{\nu, s+n+1} .
$$

Then $\left\{c_{\nu, s+i}, i=(0), 1, \ldots, n\right\}$ is the set of all the zeros inside the interval $\left[x_{\min }, x_{\max }\right]\left(c_{\nu, s}\right.$ is excluded when $\left.c_{\nu, s}<x_{\min }\right)$.

Although the forward sweep suffices to find all the zeros inside a given interval, we will prove, for completeness, that a backward method can also be built to perform this same task. No previous estimations from asymptotics are needed, similar to the forward method. The main difference between both procedures, apart from the use of the iteration $\mathcal{T}_{-\operatorname{sign}(\nu), \nu}$ instead of $\mathcal{T}_{\operatorname{sign}(\nu), \nu}$, can be found in the stopping rules which terminate the backward sweep. Particular care must be taken (rule 4 in the next theorem) in order to overcome the exception in Theorem 3.6.

Theorem 4.4 (Backward evaluation of the zeros). The zeros of any real cylinder function $\mathcal{C}_{\nu}(x)$ inside an interval $\left[x_{\min }, x_{\max }\right], x_{\min }>0$, can be evaluated in the following way:

1. Evaluate $x_{1} \equiv \lim _{m \rightarrow \infty} \mathcal{T}_{-\operatorname{sign}(\nu), \nu}^{(m)}\left(x_{\max }\right)$. Then $x_{1}=c_{\nu, s}$ for some integer $s>0$ provided there is at least one positive zero of $\mathcal{C}_{\nu}(x), c_{\nu, s^{\prime}}$, such that $c_{\nu, s^{\prime}} \leq x_{\max }$.

2. Given $c_{\nu, k}$ with $0<k \leq s$, use the backward iterative relation

$$
c_{\nu, k-1}=\lim _{m \rightarrow \infty} \mathcal{T}_{-\operatorname{sign}(\nu), \nu}^{(m)}\left(c_{\nu, k}-\pi\right)
$$

to generate the sequence of zeros

$$
c_{\nu, s}>c_{\nu, s-1}>\cdots>c_{\nu, s-n} \geq x_{\min } .
$$

3. The backward sweep must be applied reiteratively until, after the evaluation of $n$ zeros, one of the following conditions is met:

(a) $0<x_{f}<x_{\min }$, being $x_{f} \equiv \lim _{m \rightarrow \infty} \mathcal{T}_{-\operatorname{sign}(\nu), \nu}^{(m)}\left(c_{\nu, s-n}-\pi\right)$.

(b) $x_{f}=0$ ( $x_{f}$ as defined above).

(c) $c_{\nu, s-n}-\pi \leq 0$.

4. When $c_{\nu, s-n}-\pi \leq 0,|\nu|<1 / 2$ and $x_{\min }<\pi$, a further step is required. One should evaluate $x_{n+1} \equiv \lim _{m \rightarrow \infty} \mathcal{T}_{\operatorname{sign}(\nu), \nu}^{(m)}\left(x_{\min }\right)$. If $x_{\min } \leq x_{n+1}$, then $x_{n+1} \equiv c_{\nu, s-(n+1)}$ is also a zero of $C_{\nu}(x)$ in $\left[x_{\min }, x_{\max }\right]$ (the first positive zero).

$\left\{c_{\nu, s-i}, i=(0), 1, \ldots, n,(n+1)\right\}$ is the set of all the zeros inside the interval $\left[x_{\min }, x_{\max }\right] . c_{\nu, s}$ is excluded when $c_{\nu, s}>x_{\max } \cdot c_{\nu, s-(n+1)}$ is included when case 4 applies and $c_{\nu, s-n} \neq c_{\nu, s-(n+1)}$.

Remark 4.5. Of course, in a numerical implementation of the stopping rule 3(b), we would stop the backward sweep when $0<x_{f}<\epsilon, \epsilon$ being a small positive number. 
Proof of Theorems 4.3 and 4.4 . Theorems 4.3 and 4.4 follow from Theorem 3.6]and 1.13.

Given Corollary 4.2, convergence to the first zero in the interval $\left[x_{\min }, x_{\max }\right]$, $x_{\min }>0$, is guaranteed for the forward method. This fact, together with Theorems 3.6 and 1.13 proves Theorem 4.3

For the backward method, the stopping rules in Theorem4.4 steps 3, 4 remain to be proved. The situations in which the iteration must terminate are the following:

1. When $c_{\nu, 1}<x_{\min }$, the stopping rules $3(\mathrm{a})$ and $3(\mathrm{c})$ are sufficient to guarantee the proper termination of the sweep. Indeed, convergence to 0 can only take place when $B_{-\operatorname{sign}(\nu)}\left(0_{\nu}\right) \neq 0$ (Definition 1.4), that is, when $c_{\nu-\operatorname{sign}(\nu), 1}<c_{\nu, 1}$; but then, and for $s \geq 2, c_{\nu, s}-\pi$ is a starting value which guarantees convergence to $c_{\nu, s-1}$ (Theorem 3.6). Therefore, there is no possible convergence to 0 and rule 3(b) does not apply. We are left with two possibilities:

(a) The backward sweep must be halted when convergence to a zero of $\mathcal{C}_{\nu}(x)$ smaller than $x_{\min }$ takes place (stopping rule $3(\mathrm{a})$ ).

(b) When $c_{\nu, 1}<x_{\min }<c_{\nu, 2}$, it is possible that $c_{\nu, 2}-\pi \leq 0$ and then the stopping rule 3(c) applies. Notice that the algorithm is not asked to evaluate $c_{\nu, 1}$, since $c_{\nu, 1}<x_{\text {min }}$.

2. When $c_{\nu, 1} \geq x_{\min }$ the algorithm will give the set of zeros $\left\{c_{\nu, s}, s=1, \ldots, n\right\}$, where $x_{\min }<c_{\nu, 1}<c_{\nu, 2}<\cdots<c_{\nu, n}<x_{\max }$. In this case, the stopping rules become more involved, especially because the exception in Theorem 3.6 has to be resolved in order to be able to find with certainty the first positive zero $c_{\nu, 1}$ once $c_{\nu, 2}$ is known.

(a) When $c_{\nu-\operatorname{sign}(\nu), 1}<c_{\nu, 1}, c_{\nu, 2}-\pi$ is a starting value that provides convergence to $c_{\nu, 1}$ (Theorem 3.6). After the evaluation of $c_{\nu, 1}$, two different stopping rules will apply for the case $c_{\nu, 1} \leq \pi$, and for the case $c_{\nu, 1}>\pi$ :

(i) When $c_{\nu, 1}>\pi$ then necessarily $|\nu|>1 / 2$ (Lemma 4.8), and by Lemma 2.1 $\left.\left.c_{\nu, 1}-\pi \in\right] 0, c_{\nu-\operatorname{sign}(\nu), 1}\right] \equiv B_{-\operatorname{sign}(\nu)}\left(0_{\nu}\right)$; then the Newton method will converge to 0 . This situation corresponds to rule $3(\mathrm{~b})$.

(ii) When $c_{\nu, 1} \leq \pi$, the rule 3 (c) applies.

(b) When $c_{\nu, 1}<c_{\nu-\operatorname{sign}(\nu), 1}$, then $c_{\nu, 1} \leq \pi$, as will be shown in Lemma 4.7 There are two possibilities in this case:

(i) When $c_{\nu, 2}>\pi, c_{\nu, 2}-\pi$ is a starting value which guarantees convergence to $c_{\nu, 1}$ because $B_{-\operatorname{sign}(\nu)}=\emptyset$ and $0<c_{\nu, 2}-\pi<c_{\nu-\operatorname{sign}(\nu), 1}$, which means that $c_{\nu, 2}-\pi \in B_{-\operatorname{sign}(\nu)}\left(c_{\nu, 1}\right)$. This is a consequence of Lemma 3.2 for $|\nu|>1 / 2$; for $|\nu| \leq 1 / 2$ we have $c_{\nu, 2}-c_{\nu, 1} \leq \pi$ (Theorem 1.6) and then $0<c_{\nu, 2}-\pi<c_{\nu, 1}<c_{\nu-\operatorname{sign}(\nu), 1}$. After $c_{\nu, 1}$ has been evaluated, the stopping rule 3(c) terminates the sweep since $c_{\nu, 1}-\pi<0$.

(ii) If $c_{\nu, 2} \leq \pi$, the algorithm would stop just after the evaluation of $c_{\nu, 2}$ because $c_{\nu, 2}-\pi \leq 0$ (rule $3(\mathrm{c})$ ); the zero $c_{\nu, 1}$ would then be skipped. This situation constitutes the exception in Lemmas 3.33 .5 and Theorem 3.6 and it is handled and resolved by applying rule 4 (which is based on Theorem 4.2). This possibility may only be found when $|\nu|<1 / 2$ since $c_{\nu, 2} \geq \pi$ for $|\nu| \geq 1 / 2$ (Lemma 4.6).

We end this section with the proof of several results used in the preceding proof of the stopping rules $2(\mathrm{~b})$. 
Lemma 4.6. If $|\nu| \geq 1 / 2$, then $c_{\nu, 2} \geq \pi$.

Proof. The zeros of two different real cylinder functions of a same order $\nu$ are interlaced (see [26]); in particular, the zeros of $\mathcal{C}_{\nu}(x)(\alpha \neq k \pi, k \in \mathbb{Z})$ and $J_{\nu}(x)$ are interlaced; thus, $c_{\nu, 2}>j_{\nu, 1}$. Given that $j_{\nu, 1}=\pi$ and that $\frac{d j_{\nu, 1}}{d \nu}>0$ for $\nu>-1$ [26], we have that $c_{\nu, 2} \geq \pi$ for $\nu \geq 1 / 2$ and similarly (Lemma 1.8) $c_{\nu, 2} \geq \pi$ for $\nu \leq-1 / 2$.

Lemma 4.7. If $c_{\nu, 1}<c_{\nu-\operatorname{sign}(\nu), 1}$, then $|\nu|<1, \nu \neq 0$ and $c_{\nu, 1} \leq \pi$.

Proof. Given Theorem 4.1, $c_{\nu, 1}<c_{\nu-\operatorname{sign}(\nu), 1}$ cannot take place when $|\nu|>1$ or $\nu=0$.

Let us consider $\nu \in] 0,1\left[\right.$ to show that $c_{\nu, 1} \leq \pi$; then the use of Lemma 1.8 guarantees that the same inequality holds for $\nu \in]-1,0[$.

Since $y_{\nu, 1}<j_{\nu, 1}<y_{\nu, 2}<j_{\nu, 2}<\ldots$ for $\nu>0$, where $y_{\nu, s}$ and $j_{\nu, s}$ are the $s$ th positive zeros of $J_{\nu}(x)$ and $Y_{\nu}(x)$, respectively, and given that for $x \rightarrow 0^{+}$

$$
J_{\nu}\left(0^{+}\right)=0^{+}, J_{\nu}^{\prime}\left(0^{+}\right)>0, Y_{\nu}\left(0^{+}\right)<0,
$$

we have that

1. $\mathcal{C}_{\nu}\left(y_{\nu, 1}\right)>0$ and $\mathcal{C}_{\nu}\left(j_{\nu, 1}\right)<0$ for $\left.\alpha \in\right] 0, \pi / 2\left[\right.$. Then $c_{\nu, 1} \in\left[y_{\nu, 1}, j_{\nu, 1}\right]$ for $\alpha \in[0, \pi / 2]$ by Rolle's theorem.

2. $\mathcal{C}_{\nu}(0+)>0$ and $\mathcal{C}_{\nu}\left(y_{\nu, 1}\right)<0$ for $\left.\alpha \in\right] \pi / 2, \pi\left[\right.$. Then $\left.c_{\nu, 1} \in\right] 0, y_{\nu, 1}[$, where $\mathcal{C}_{\nu}(x)=\cos \alpha J_{\nu}(x)-\sin \alpha Y_{\nu}(x)$.

Thus,

$$
c_{\nu, 1} \leq j_{\nu, 1} \quad \forall \alpha .
$$

Besides, since $\mathcal{C}_{\nu-1}(x)=-\cos (\alpha+\nu \pi) J_{1-\nu}(x)+s(\alpha+\nu \pi) Y_{1-\nu}(x)$ (Lemma 1.8), by repeating the same argument we have that $\left.c_{\nu-1,1} \leq j_{1-\nu, 1}, \nu \in\right] 0,1[$. But our hypothesis is $c_{\nu, 1}<c_{\nu-1,1}$ for $\nu>0$ and then

$$
\left.c_{\nu, 1}<j_{1-\nu, 1} \quad \forall \alpha \forall \nu \in\right] 0,1[.
$$

Equations 4.2 and 4.3 together with the fact that $d j_{\nu, 1} / d \nu>0$ for $\nu>-1$ [26] leads to

$$
c_{\nu, 1} \leq j_{1 / 2,1}=\pi
$$

Lemma 4.8. If $|\nu| \leq 1 / 2$, then $c_{\nu, 1} \leq \pi$.

Proof. Similar to Lemma 4.7] $c_{\nu, 1} \leq j_{\nu, 1}$ and then $c_{\nu, 1} \leq \pi$.

\section{NUMERICAL EXAMPLES}

In this section, several examples of the application of Lemmas 4.3 and 4.4 are provided. Both the forward and backward sweeps have been proven to be capable of obtaining all the real zeros of any real cylinder function. Therefore, a sufficiently accurate method of evaluation of cylinder functions is all that is needed to evaluate their zeros.

As discussed in [20] and [21], a continued fraction (CF) can be used for first kind Bessel functions to evaluate $J_{\nu}(x) / J_{\nu+i}(x), i= \pm 1$. The use of the CF gives rise to a short and accurate method of computation.

To obtain the zeros of combinations $\cos \alpha J_{\nu}(x)-\sin \alpha Y_{\nu}(x)$ with $\alpha \neq 0$, a continued fraction is no longer available since the resulting cylinder function is 
no longer minimal with respect to recursion over orders. However, we can evaluate both $J_{\nu}(x)$ and $Y_{\nu}(x)$ by using some external program. We use SLATEC routines DBESJ and DBESY to evaluate $J_{\nu}(x)$ and $Y_{\nu}(x)$ for $\nu \geq 0$, and we apply Lemma 1.8 for $\nu<0$.

Overflows in the evaluation of cylinder functions can be expected. The main problem is due to the fact that $Y_{\nu}(x)$ goes to $-\infty$ as $x \rightarrow 0^{+}$. This behaviour causes rounding problems when trying to obtain the first positive zero for $\nu>0$ and $\alpha=k \pi-\epsilon$ with $0<\epsilon \ll 1\left(c_{\nu, 1} \rightarrow 0^{+}\right.$as $\left.\epsilon \rightarrow 0^{+}\right)$. For $\nu<0$ the same cancellation problems appear when $-\nu \pi+\alpha=k \pi-\epsilon, 0<\epsilon \ll 1$. This limitation is difficult to overcome and was also present in the evaluation of the zeros of $J_{\nu}(x)$ 21] for negative orders $\nu=-k+\epsilon$.

In addition, one has to detect and avoid overflows in the ratio $\mathcal{C}_{\nu}(x) / \mathcal{C}_{\nu+i}(x)$ $(i= \pm 1)$ when $x$ is close to a zero of $\mathcal{C}_{\nu+i}(x)$. As discussed in [20] and [21, the use of the Lentz-Thompson algorithm [19] 24] to evaluate the CF for $J_{\nu}(x) / J_{\nu \pm 1}(x)$ overcomes this problem while, for general combinations, one has to take explicit precautions. This overflow problem can be handled with ease.

Apart from the roundoff error limitations, to evaluate the first positive zeros in the cases when $c_{\nu, 1}$ is small, the application of Theorems 4.3 and 4.4 provides a general algorithm to find all real roots of any real cylinder function. As an illustration, the zeros for $\mathcal{C}_{\nu}(x)$ for $\nu=0.2$ and $\nu=10$ and $\alpha$ varying in $[0, \pi[$ (taking steps $\Delta \alpha=0.1$ ) are shown in Figure 1. We also show the zeros of the contiguous cylinder functions $\mathcal{C}_{\nu \pm 1}(x)$ and starting values used both by the forward and backward algorithms.

The left figure shows the zeros found in the interval $[15,35]$ for $\mathcal{C}_{\nu}(x)$ with $\nu=$ $10,9,11$ and $\alpha \in[0, \pi[$. In this figure, the smallest zeros are the first positive zeros. Starting values used to evaluate the zeros of $\mathcal{C}_{10}(x)$, for both the forward and backward sweep are shown ( $\times$-points).

Note that for a fixed value of $\alpha$ the starting values for the backward iteration always lie between two consecutive zeros of $\mathcal{C}_{9}(x)$ ( $\square$-points) which are adjacent to the zero of $\mathcal{C}_{10}(x)$ to which the Newton iteration converges (Theorem 1.13). The last starting value (the lowest) is such that the Newton iteration would converge to zero, which is the situation corresponding to the stopping rule 3(b) of Theorem 4.4.

On the other hand, the starting values for the forward iteration always lie between two consecutive zeros of $\mathcal{C}_{11}(x)$ (+-points) which are adjacent to a same zero of $\mathcal{C}_{10}(x)$. The last (highest) starting value lies outside the interval $[15,35]$ and gives convergence to a zero outside this interval (stopping rule of Theorem 4.3).

A graphical explanation for the different Newton iterations used by the forward and backward sweeps can be given by considering Figure 1 (left). Given two consecutive starting values for the backward sweep there is one and only one curve of $\square$-points (zeros of $\mathcal{C}_{9}(x)$ ) crossing between both starting values. In addition, between each two consecutive starting values for the forward sweep there is one and only one curve of + -points crossing (zeros of $\mathcal{C}_{11}(x)$ ). This property shows that two consecutive starting values always lie in consecutive (and different) branches $B_{\operatorname{sign}(i \nu)}\left(c_{\nu, s}\right), B_{\operatorname{sign}(i \nu)}\left(c_{\nu, s \pm 1}\right)(i=+1$ for the forward sweep, $i=-1$ for the backward case). However, we see in Figure 1 (left) that there is a case in which two lines of $\square$-points cross between two consecutive starting values for the forward sweep; one can also observe that there are two consecutive starting values for the backward 

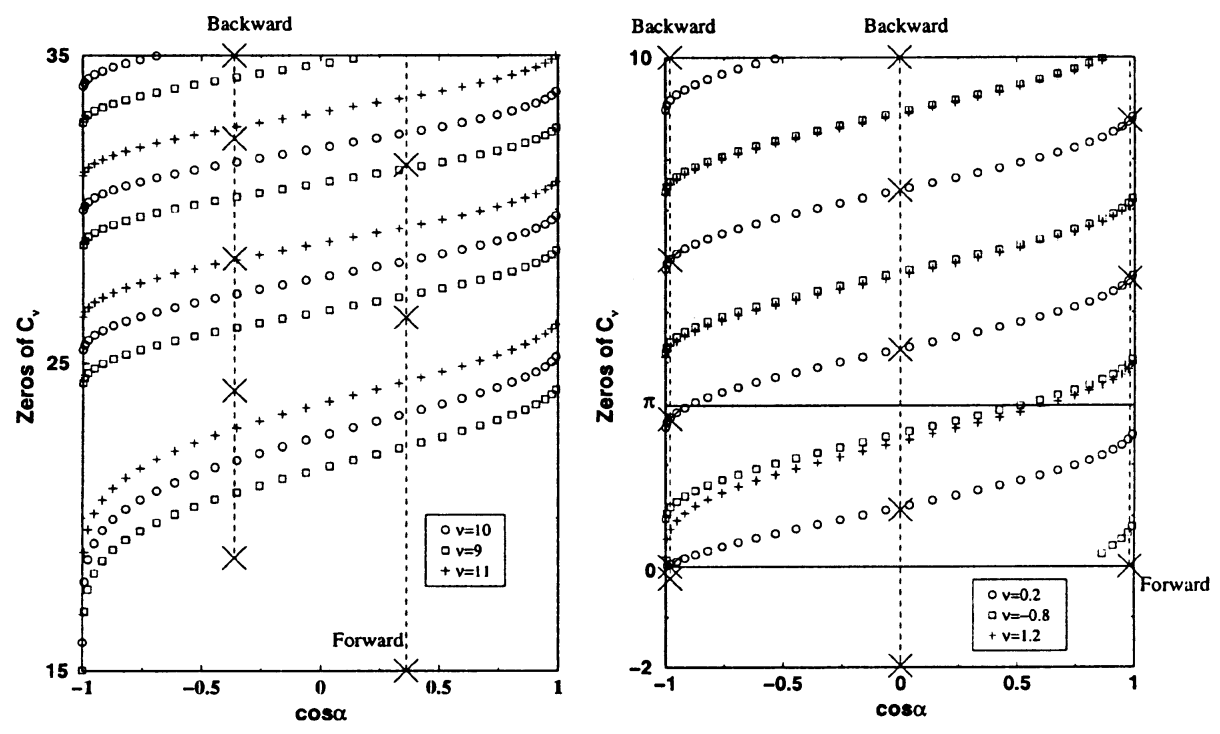

Figure 1. First positive zeros of the cylinder functions $\mathcal{C}_{\nu}(x)=$ $\cos \alpha J_{\nu}(x)-\sin \alpha Y_{\nu}(x)$ for $\nu=10$ (left) and $\nu=0.2$ (right) and $\alpha \in\left[0, \pi\left[\right.\right.$. The zeros for the contiguous cylinder functions $\mathcal{C}_{\nu \pm 1}$ and the starting values considered to evaluate several zeros $c_{\nu, s}$ are also shown.

sweep which are not separated by any curve of +-points. This fact explains graphically why two different Newton iterations are needed depending upon the type of sweep considered (forward/backward).

The right figure shows the first zeros for the orders $\nu=0.2,-0.8,1.2$ within the interval $[0.001,10]$. The most interesting differences with respect to the right figure can be found in the stopping rules for the backward iteration.

The starting values for the forward sweep guarantee convergence to all zeros in the interval $[0.001,10]$. The finding of zeros stops when a zero larger than $x_{\max }=10$ is found.

In a similar way, all the starting values for $\cos (\alpha)=0$ used by the backward sweep guarantee, without exception, convergence to all positive zeros in the interval $[0.001,10]$. The last starting value is negative given that $c_{\nu, 1}<\pi$ (stopping rule 3 (c) of Theorem 4.4).

Finally, the starting values for the backward algorithm when $\cos \alpha \simeq-1$, illustrate the exception to Theorem 3.6 and the necessity of stopping rule 4 in Theorem 4.4. Observe that $0 \simeq c_{\nu, 1}<c_{\nu, 2}<\pi$ so that, after $c_{\nu, 2}$ has been evaluated, the new starting value $c_{\nu, 2}-\pi$ is smaller than zero. The algorithm stops due to rule 3(c) but the first zero can be evaluated a posteriori by using rule 4 with $x_{\min }=0.001$.

\section{Conclusions}

Two global Newton iterations have been built, based on the monotonic functions $f_{ \pm 1, \nu}(x)$. This pair of global Newton iterations (Theorem 1.13), complemented with the bounds on differences of adjacent zeros (Theorem 3.2), enabled us to find forward and backward iterative relations (Theorem [3.6) between consecutive zeros 
of general cylinder functions $\mathcal{C}_{\nu}(x)$. By using these iterative relations all the zeros of any real cylinder function inside a given interval $\left[x_{\min }, x_{\max }\right], x_{\min }>0$, can be found. Forward or backward sweeps have been built which are able to perform this calculation (Theorems 4.3 and 4.4).

These global methods are expected to be particularly efficient for the evaluation of the smallest zeros for low $|\nu|$ (as happened for $J_{\nu}(x)$ 21]), precisely when asymptotic expansions tend to fail. Furthermore, the method presented here is valid for any real cylinder function of any real order.

\section{ACKNOWLEDGMENT}

The author thanks A. Gil for carefully reading the manuscript.

\section{REFERENCES}

1. M. Abramowitz and I.A. Stegun (editors), Handbook of Mathematical Functions, Dover Publications Inc. (1972). MR 94b:00012

2. C. Belingeri, P.E. Ricci, "On asymptotic formulas for the first zero of the Bessel function $J_{\nu}(x) "$, J. Inf. Optimization. Sci. 17 (1996) 267-274. MR 98c:33004

3. Ll. G. Chambers, "An upper bound for the first zero of the Bessel function $J_{\nu}$ ", Math. Comp. 38 (1982) 589-591. MR 83h:33011

4. Á. Elbert, A. Laforgia, "On the convexity of the zeros of Bessel functions", SIAM J. Math. Anal. 16 (1985) 614-619. MR 86g:33008

5. Á. Elbert, "An approximation for the zeros of Bessel functions", Numer. Math. 59 (1991) 647-657. MR 92h:33008

6. Á Elbert, A. Laforgia, "An upper bound for the zeros of the cylinder function $\mathcal{C}_{\nu}(x)$ ". Math. Inequal. Appl. 1 (1998) 105-111. MR 99c:33005

7. E.K. Ifantis, P.D. Siafarikas, "A differential inequality for the positive zeros of Bessel functions", J. Comput. Appl. Math. 44 (1992) 115-120. MR 94a:33003

8. K.S. Kölbig, CERNLIB-Short Writeups, subroutine DBZEJY(C345), library MATHLIB http://wwwcn.cern.ch/shortwrupsdir/index.html.

9. P. Kravanja, O. Ragos, M. N. Vrahatis, F.A. Zafiropoulos. "ZEBEC: A mathematical software package for computing simple zeros of Bessel functions of real order and complex argument". Comput. Phys. Commun. 113 (1998) 220-238.

10. N.N. Lebedev, Special Functions and their Applications, Dover Publications Inc. (1972). MR 50:2568

11. Y. L. Luke, Mathematical functions and their approximations, Academic Press, New York, 1975. MR 58:19039

12. L. Lorch, R. Uberti, " "Best possible" upper bounds for the first positive zeros of Bessel functions - the finite part", J. Comput. Appl. Math. 75 (1996) 249-258. MR 98b:33010

13. M. E. Muldoon, "Convexity properties of special functions and their zeros" in Recent Progress in Inequalities, pp. 309-323, Kluwer Academics Publishers, Dordrecht-Boston-London (1998). CMP 98:11

14. F.W.J. Olver (editor), Royal Society Mathematical Tables, Bessel functions, Part III. Zeros and Associated Values, vol. 7, Cambridge University Press, London-New York, 1960. MR 22:10202

15. R. Piessens, "Rational approximations for the zeros of Bessel functions", J. Comput. Phys. 42 (1981) 403-405.

16. R. Piessens, "Chebyshev series approximations for the zeros of Bessel functions", J. Comput. Phys. 53 (1984) 188-192. MR 85h:65044

17. R. Piessens, "A series expansion for the first positive zero of the Bessel functions, Math. Comp. 42 (1984) 195-197. MR 84m:33014

18. R. Piessens, "On the computation of zeros and turning points of Bessel functions, Bull. Greek Math. Soc. 31 (1990) 117-122. MR 92e:65019

19. W.H. Press, S.A. Teukolsky, W.T. Vetterling and B.P. Flannery, Numerical Recipes in Fortran. Cambridge University Press (1992). MR 93i:65001a 
20. J. Segura, "A global Newton method for the zeros of cylinder functions", Num. Algorithms 18 (1998) 259-276. MR 99h:65092

21. J. Segura \& A. Gil, "ELF and GNOME: Two tiny codes to evaluate the real zeros of the Bessel functions of the first kind for real orders", Comput. Phys. Commun. 117 (1999) 250. CMP 99:10

22. N.M. Temme, "An algorithm with ALGOL 60 program for the computation of the zeros of ordinary Bessel functions and those of their derivatives". J. Comp. Phys. 32 (1979) 270-279.

23. N.M. Temme, Special Functions: an introduction to the Classical Functions of Mathematical Physics. John Wiley \& Sons (1996). MR 97e:33002

24. I.J. Thompson and A.R. Barnett, "Coulomb and Bessel functions of complex arguments and order". J. Comput. Phys. 64 (1986) 490-509. MR 87h:33012

25. M.N. Vrahatis, O. Ragos, T. Skiniotis, F.A. Zafiropoulos, T.N. Grapsa. "RFSFNS: A portable package for the numerical determination of the number and the calculation of roots of Bessel functions. Comput. Phys. Commun., 92 (1995) 252-266.

26. G. N. Watson, A Treatise on the Theory of Bessel Functions, Cambridge University Press, London (1944). MR 6:64a

Instituto de Bioingeniería, Universidad Miguel Hernández, Edificio La Galia, 03202Elche, Alicante, Spain

E-mail address: segura@flamenco.ific.uv.es, javi.segura@umh.es 